\title{
Programmaufwand, Qualität und Wirtschaftlichkeit öffentlich-rechtlicher Rundfunkangebote
}

\author{
Wolfgang Seufert
}

Betriebswirtschaftliche Kennziffern bzw. Indikatoren dienen sowobl der internen Steuerung von Produktionsprozessen als auch der externen Kontrolle der Zielerreichung durch die Aufsichtsgremien von Unternehmen. Der Beitrag befasst sich mit zwei Aspekten der externen Kontrolle der Wirtschaftlichkeit öffentlich-rechtlicher Rundfunkanstalten: mit der Frage, ob der von der KEF verwendete Indikator „Minutenkosten “ für solche Wirtschaftlichkeitsvergleiche generell geeignet ist, und mit der Frage, inwieweit das Kostenniveau privater Anbieter auch als Zielwert (Benchmark) für öffentlich-rechtliche Angebote herangezogen werden kann. Auf Basis theoretischer Überlegungen zum Zusammenhang zwischen Programmaufwand, Qualität und Zuschauer-bzw. Hörernachfrage wird deutlich, dass Tausenderkontaktkosten (TKK) als Wirtschaftlichkeitsindikator für Rundfunkveranstalter generell besser geeignet sind als Minutenkosten, wobei zu berücksichtigen ist, dass sich das TKK-Niveau für unterschiedliche Programmgenres systematisch unterscheidet. Unterschiedliche Programmstrukturen bzw. spezielle Vorgaben für die öffentlich-rechtlichen Angebote (z. B. für Informations- oder Wortanteile) schlagen deshalb auf deren TKK-Niveau durch. In einem empirischen Teil werden für die deutschen TV-Vollprogramme und landesweiten Hörfunkprogramme die vermuteten Zusammenhänge zwischen Kostenniveau und Rezipientennachfrage bzw. zwischen Produktionsaufwand und Programmstruktur bestätigt. Private TKK-Niveaus eignen sich als Benchmark nur eingeschränkt, da bierbei auch die Effekte von Marktanteilszielen als einer weiteren Qualitätsdimension öffentlich-rechtlicher Angebote zu berücksichtigen sind.

Schlagwörter: Medienqualität, Produktionsaufwand, Rundfunkfinanzierung, Rundfunkregulierung, Tausenderkontaktkosten, Wirtschaftlichkeit

\section{Minutenkosten als Indikator für die Wirtschaftlichkeit von Rundfunkangeboten?}

Mit dem Übergang zum dualen Rundfunksystem im Jahr 1984 hat sich die Diskussion über die wirtschaftliche Effizienz bzw. Wirtschaftlichkeit des öffentlich-rechtlichen Rundfunks insofern verändert, als sie verstärkt unter Bezugnahme auf das Gegenmodell „privater Rundfunk“ erfolgt (u. a. Kronberger Kreis, 1989; Heinrich, 2005). Dabei wird dem öffentlich-rechtlichen Teilsystem häufig pauschal unwirtschaftliches Verhalten vorgeworfen und der private Rundfunk generell als effizienter und kostengünstiger eingeschätzt (u. a. Eickhof/Never, 2000; Radke/Then Berg, 2004), wobei als empirischer Beleg niedrigere Durchschnittskosten der privaten Anbieter angeführt werden. Immer häufiger versucht die Medienpolitik deshalb - zuletzt durch eine willkürliche Begrenzung des von der KEF empfohlenen Rundfunkgebührenanstiegs - einen Sparzwang auf die öffentlich-rechtlichen Rundfunkanstalten auszuüben. Am weitesten geht bislang das Positionspapier der Ministerpräsidenten von Bayern, Nordrhein-Westfalen und Sachsen, in dem 2003 konkrete Sparsummen und Programmreduzierungen gefordert wurden (Gemeinsame Initiative der Bundesländer Bayern, NRW und Sachsen, 2003).

Aus einer medienökonomischen Perspektive ist dabei überraschend, dass diese Aus- 
einandersetzung über die Wirtschaftlichkeit des öffentlich-rechtlichen Rundfunks fast keinen Bezug zur Qualitätsdebatte hat, in der wiederum der Vorwurf der Selbstkommerzialisierung, d. h. einer zu starken Anpassung der öffentlich-rechtlichen Programme an das niedrigere - weil am Massengeschmack ausgerichtete - Qualitätsniveau der Privaten dominiert (vgl. Krüger, 1991; Heyen, 2001; Stock, 2005). Auch bei der Produktion von TV- und Radioprogrammen ist aber - wie in allen anderen Wirtschaftszweigen - davon auszugehen, dass ein Zusammenhang zwischen Produktqualität und Produktionsaufwand besteht, höhere Qualitätsansprüche sich in der Regel also in einem höheren Kostenniveau widerspiegeln.

Die Ausblendung des Zusammenhangs von Kosten und Qualität ist sicher auch auf die Praxis der Kommission zur Ermittlung des Finanzbedarfs der Rundfunkanstalten (KEF) in ihren Wirtschaftlichkeitsvergleichen zwischen den öffentlich-rechtlichen Rundfunkanstalten zurückzuführen. Diese werden im Auftrag der Ministerpräsidenten der Länder seit 1991 regelmäßig durchgeführt (vgl. Nafziger/Schwertzel, 1996) und nutzen als wichtigste Vergleichsgröße die jeweiligen durchschnittlichen Minutenkosten der gesendeten Programmminuten (zuletzt KEF, 2005: 27ff.). Das Kostenniveau der jeweils günstigsten Anstalt soll dabei als Benchmark für Einsparpotenziale bei den anderen Anstalten dienen (KEF, 2003: 251). Damit wird jedoch eine Kennziffer verwendet, mit der Qualitätsunterschiede von Rundfunkprogrammen nicht angemessen berücksichtigt werden können. Der folgende Beitrag untersucht deshalb zwei Fragen:

Gibt es für einen Wirtschaftlichkeitsvergleich öffentlich-rechtlicher Rundfunkangebote eine andere Kennziffer als die „durchschnittlichen Minutenkosten“, die die Interdependenz von Qualitätszielen und Wirtschaftlichkeitszielen besser erfasst?

Eignet sich ein solcher Indikator damit auch für einen Wirtschaftlichkeitsvergleich zwischen öffentlich-rechtlichen Anstalten und privaten Anbietern, indem er die spezifischen Qualitätsziele öffentlich-rechtlicher Rundfunkangebote mit berücksichtigen kann?

\section{Wirtschaftlichkeitskennziffern für Rundfunkprogramme - Minutenkosten versus Tausenderkontaktkosten (TKK)}

\subsection{Stückkosten als Wirtschaftlichkeitskennziffern}

Auch Non-Profit-Unternehmen werden von ihren Eigentümern in der Regel zu wirtschaftlichem Verhalten verpflichtet, damit sie ihre gemeinnützigen Zwecke bestmöglich erfüllen können. Dabei kann zwei Entscheidungsregeln gefolgt werden. Wird Kostenwirksamkeit angestrebt, soll mit den vorhandenen Ressourcen eine maximale Leistung, meist definiert über Outputmengen und Qualitätsniveaus, erzielt werden (Maximalprinzip). Wird Kostenwirtschaftlichkeit angestrebt, soll eine definierte Leistung mit minimalem Ressourceneinsatz erreicht werden (Minimalprinzip). In beiden Fällen geht es also darum, das Verhältnis von Leistungs- bzw. Outputeinheiten zu Inputeinheiten zu optimieren (Nafziger/Schwerzel, 1996: 21; Schumann/Hess, 2003: 3).

Um der Unternehmensleitung und den Mitarbeitern intern ökonomisch rationale Produktionsentscheidungen zu ermöglichen und um darüber hinaus auch eine effiziente externe Kontrolle durchführen zu können, müssen Produktionsmenge, Produktqualität und Inputs durch geeignete Kennziffern bzw. Indikatoren abgebildet werden. Grundsätzlich kann dabei zur Quantifizierung der Input/Outputrelationen mit Kennziffern unterschiedlicher Dimensionen gearbeitet werden: 
- Faktorproduktivitäten setzen die Leistungs- bzw. Outputeinheiten mit den notwendigen Inputmengen (z. B. Arbeitsstunden) ins Verhältnis.

- Stückkosten setzen Leistungs- bzw. Outputeinheiten - bei gegebenen Preisen für die Produktionsfaktoren - zu den Inputwerten (z. B. Lohnkosten) ins Verhältnis.

Während hierbei gewinnorientierte Unternehmen versuchen, ihre Profitrate zu maximieren und eine möglichst große positive Differenz zwischen dem Preis einer Outputeinheit und ihren Stückkosten zu erreichen, gilt bei Non-Profit-Unternehmen das Kostendeckungsprinzip.

\subsection{Leistungseinheit Programmmenge: Minutenkosten als Wirtschaftlichkeitsindikator}

Zur Berechnung von Stückkosten bietet es sich auf den ersten Blick an, die Outputeinheiten von TV- und Radioprogrammen als Zeitmengen zu definieren, beispielsweise als Zahl der eigenproduzierten oder der gesendeten Programmminuten. Allerdings spricht dagegen, dass es sich bei Rundfunkprogrammen nicht um standardisierbare Güter handelt. Sie bestehen, sieht man von Wiederholungen ab, aus Einzelanfertigungen (Unikate). Diese Einzelbeiträge bzw. Sendungen können zudem unterschiedlich zu Programmen kombiniert werden. Rundfunkprogramme sind also sehr heterogene Leistungsbündel. Im Ergebnis variieren die Stückkosten auf der Basis von Minutenkosten ebenso stark zwischen den Sendungen und Einzelbeiträgen eines Veranstalters wie zwischen den Gesamtangeboten verschiedener TV- oder Radioveranstalter.

Die KEF versucht diesem Problem zu begegnen, indem sie ihre Kostenvergleiche auf Basis von Minutenkosten nicht allein für das jeweilige Gesamtprogramm der Anstalten, sondern auch für Programmgenres, d. h. für inhaltlich ähnliche Sendungen, durchführt. Im 15. KEF-Bericht werden beispielsweise die TV-Minutenkosten der Dritten Programme im Jahr 2004 in acht Kategorien („Politik und Gesellschaft“, „Kultur und Wissenschaft“, „Sport“, „Fernsehspiel“, Spielfilm“, „Unterhaltung“, „Familie“ sowie „Bildung und Beratung") differenziert. Die Minutenkostenvergleiche der ARD-Radioprogramme beziehen sich auf drei Wortkategorien („Information und Service“, „Kultur/Bildung“, „Unterhaltung“) sowie drei Musikkategorien („Rock-/Popmusik“, „Unterhaltungsmusik“, „Klassik“).

\subsection{Leistungseinheit Zuschanermenge: Tausenderkontaktkosten (TKK) als Wirtschaftlichkeitsindikator}

Selbst bei ausreichend tiefer Genredifferenzierung stellt sich allerdings grundsätzlich die Frage, ob die Leistungseinheit „Sendeminuten“- die für Rundfunkproduktionsbetriebe sinnvoll sein mag - für TV- und Radioveranstalter ebenfalls geeignet ist.

Für einen privaten TV-Veranstalter ist es beispielsweise keine sinnvolle Strategie, ein Programm mit minimalen Minutenkosten zu produzieren, indem er sein Leistungsbündel aus möglichst kostengünstigen Programmelementen zusammenstellt. Gesetzt den Fall, Unterhaltungsangebote hätten im Durchschnitt doppelt so hohe Minutenkosten wie Informationsangebote, aber ein dreimal so hohes Zuschauerpotenzial, dann würde ein Anbieter konsequenterweise auf die Ausstrahlung der „billigeren“ Informationssendungen verzichten und nur die nach diesem Maßstab „teureren“ Unterhaltungsprogramme senden.

Für ein gewinnorientiertes Rundfunkunternehmen ist es also rationaler, die Rentabilität bzw. Wirtschaftlichkeit von Programmalternativen nicht auf der Basis der Minutenkosten zu vergleichen, sondern auf Basis von Tausenderkontaktkosten (TKK), bei 
denen die Minutenkosten zusätzlich auf jeweils 1000 erreichte Zuschauer oder Hörer bezogen werden. ${ }^{1}$

Dies bedeutet aber nicht, dass alle privaten Anbieter deshalb das gleiche „quotenmaximierende" Programmbündel anbieten und keine kleineren Rezipientengruppen mit speziellen Programminteressen mehr bedienen werden. Allerdings werden auf Dauer nur Genres in das Angebot aufgenommen werden, die auf eine ausreichend große Nachfrage stoßen. Aus einer mikroökonomischen Perspektive haben solche rentablen Angebote damit per definitionem auch eine ausreichende Mindestqualität. Danach gibt es zwar in der Regel bei allen Gütern sowohl objektiv messbare als auch subjektiv wahrgenommene Qualitätsunterschiede - normativ ist dabei jedoch eine „objektiv höhere“ Qualität der „objektiv niedrigeren“ Qualität nicht vorzuziehen. Solange Qualitätsmängel keine nachhaltigen Schäden bei den Nutzern (oder negative externe Effekte für Dritte) verursachen, zählt allein das vom subjektiven Nutzen der Rezipienten abhängige "gewünschte“" Qualitätsniveau. ${ }^{2}$

\subsection{TKK für Gesamtprogramme versus TKK für Programmgenres}

Inwieweit sind Wirtschaftlichkeitsvergleiche auf der Basis von Tausenderkontaktkosten aber auch für öffentlich-rechtliche Rundfunkveranstalter sinnvoll, die ja besonderen gesetzlichen Qualitätsanforderungen unterliegen und für die deshalb nicht die Option besteht, ihr Programmangebot völlig frei zu gestalten und allein auf eine Maximierung der Zuschauer- bzw. Hörerzahlen auszurichten?

In der aktuellen Fassung des Rundfunkstaatsvertrags (Achter Rundfunkänderungsstaatsvertrag) sind im Paragraph 11 die Aufgaben der Rundfunkanstalten festgelegt: eine Pflicht zur umfassenden und ausgewogenen Informationsvermittlung, zur Sendung von Bildungsangeboten sowie von qualitativ hochwertigen Kultur- und Unterhaltungsprogrammen. Eingeschlossen sind hierin nach überwiegender Ansicht auch so genannte Minderheitenprogramme, die nach rein kommerziellen Gesichtspunkten aufgrund der geringen Rezipientenzahlen nicht rentabel wären, von denen aber angenommen wird, dass sie unter anderem zur gesellschaftlichen Integration sozialer und kultureller Minderheiten beitragen (u. a. Eifert/Hoffmann-Riem, 1999; Holznagel/Vesting, 1999; Ladeur, 2000; Brenner, 2002).

Die öffentlich-rechtliche Programmqualität wird deshalb in der öffentlichen Debatte auch meist anhand der Programmstruktur bewertet (u. a. Kruse, 2004), insbesondere als explizite oder implizite Zielvorgabe für den Anteil an Informations-, Bildungs- und Kultursendungen im TV-Programm oder für den Wortanteil im Radioprogramm. Die unterstellte positive gesellschaftliche Wirkung der Nutzung solcher Programmgenres kann allerdings nur eintreten, wenn derartige Sendungen auch rezipiert werden. Die Reichweite stellt damit zwar nicht für das gesamte öffentlich-rechtliche Angebot, wohl aber für diese Programmteile eine notwendige weitere Qualitätsdimension dar (vgl. Ha-

1 Dies entspricht der Berechnung der Werbepreise als Tausender-Kontakt-Preise (TKP). Um das Preisniveau für die Ausstrahlung eines Werbespots mit einer bestimmten Dauer zwischen verschiedenen Anbietern vergleichen zu können, werden diese von den Werbetreibenden als TKP berechnet, in der Regel wird der Preis je 30 Werbesekunden auf je 1000 erzielbare Werbekontakte bezogen (Siegert/Brecheis, 2005: 199).

2 Ein hoher Marktanteil bzw. eine hohe „Quote“ eines Programms ist damit auch nicht zwingend ein Beleg für eine hohe Qualität, sondern zeigt zunächst nur, wie groß das Nachfragesegment für einen bestimmten Qualitätsstandard ist. 
sebrink, 2005: 453). Insofern sind die Tausenderkontaktkosten (TKK) als Wirtschaftlichkeitsindikator auch für Vergleiche zwischen öffentlich-rechtlichen Anbietern grundsätzlich besser geeignet als Minutenkosten.

Ein Wirtschaftlichkeitsvergleich zwischen öffentlich-rechtlichen und privaten Anbietern auf Basis der TKK für Gesamtprogramme würde aufgrund der unterschiedlichen Programmstrukturen allerdings nicht unbedingt viel bessere Ergebnisse liefern als ein Vergleich auf Basis von Minutenkosten. Anders sähe es aus, wenn man solche Wirtschaftlichkeitsvergleiche nur auf ähnliche Programmelemente beschränken würde, sie also auf Basis von TKK für Programmgenres durchführt. Die Programmgenres sollten dabei aus theoretischer Sicht idealerweise so abgegrenzt sein, dass sie einerseits Angebote mit möglichst homogenen Produktionsprozessen darstellen und andererseits auch unterschiedliche Nachfragepotenziale abbilden, aus Rezipientenperspektive also echte Teilmärkte (Marktsegmente) darstellen. ${ }^{3}$

\section{Theoretischer Zusammenhang zwischen Produktionsaufwand und Rezipientennachfrage}

\subsection{Qualitätswettbewerb zwischen Rundfunkanbietern und "Qualitätsoptimum"}

Die in der Mikroökonomie vorherrschende Vorstellung vom Wettbewerb zwischen Unternehmen geht von einem Kostensenkungswettbewerb aus, der in einen Preiswettbewerb übergeht. Der einzelne Anbieter versucht, durch Produktivitätsfortschritte kostengünstiger zu produzieren als die Konkurrenten und dann über Preissenkungen höhere Marktanteile zu gewinnen. Die Konkurrenten können entweder bei den Produktivitätsfortschritten nachziehen, oder sie scheiden langfristig wegen mangelnder Rentabilität aus dem Markt aus.

$\mathrm{Da}$ es sich bei Medienprodukten um Einzelanfertigungen (Unikate) handelt, wird die Nachfrageentscheidung der Rezipienten in der Regel stärker von den Produktmerkmalen (ihren qualitativen Eigenschaften) beeinflusst als vom Preis. Zudem werden die meisten privaten Rundfunkprogramme ausschließlich oder überwiegend über Werbung finanziert, da sich damit die notwendigen Transaktionskosten für den Ausschluss von Nichtzahlen im Vergleich zu Pay-Angeboten stark reduzieren lassen (Picard, 1989: 28). Ein Preiswettbewerb macht auf Rundfunkmärkten also kaum Sinn. Umstritten ist, ob es trotzdem zu einer Kostensenkungsstrategie mit in der Tendenz negativen Auswirkungen auf den Qualitätsstandard von TV- und Radioprogrammen kommt (Heinrich, 1996), oder ob der Wettbewerb zwischen den Rundfunkanbietern nicht im Gegenteil über eine Erhöhung der Produktqualitäten erfolgt, der so im Zeitverlauf eher zu einer Anhebung des Kostenniveaus führt (Seufert, 1992).

Das Hauptargument für die Kostensenkungstendenz ist die asymmetrische Informationsverteilung zwischen Anbietern und Rezipienten in Bezug auf die Produktqualität.

3 Bei der Abgrenzung von Märkten bzw. Teilmärkten wird in der ökonomischen Wettbewerbstheorie nicht von objektiven Produkteigenschaften, sondern vom Prinzip der funktionalen Äquivalenz ausgegangen (Bedarfsmarktkonzept). Zu einem Markt werden danach alle Produkte gerechnet, die aus Sicht der Nachfrager funktional austauschbar sind und so wechselseitig ein hohes Substitutionspotenzial besitzen (Czygan/Kallfaß, 2003: 299). Übertragen auf TVProgramme wäre danach das Substitutionspotenzial zwischen unterschiedlichen Qualitätsstufen eines Programmgenres also höher als zwischen verschiedenen Programmgenres, weil diese definitionsgemäß unterschiedliche Rezipientenbedürfnisse befriedigen. 
Insbesondere bei Informationsangeboten kann ihr Wahrheitsgehalt selbst nach ihrem „Konsum“ nicht angemessen beurteilt werden. Für die Produzenten gibt es deshalb keinen Anreiz, einen im Vergleich zur Konkurrenz höheren Rechercheaufwand oder andere teure und qualitätssteigernde Maßnahmen zu ergreifen, wenn diese von den Rezipienten nicht wahrgenommen werden können. Es kommt damit tendenziell zu einem Marktgleichgewicht, bei dem alle Anbieter auf einem Mindestqualitätsniveau produzieren (Lobigs, 2004).

Die Gegenposition eines kostensteigernden Qualitätswettbewerbs geht damit von zwei impliziten Voraussetzungen aus: zum einen, dass der Rezipient beim überwiegenden Teil der angebotenen Rundfunkprogramme einen höheren Produktionsaufwand auch als höheren Qualitätsstandard wahrnimmt, und zum anderen, dass er Angebote mit höherer Qualität denen mit niedrigerer Qualität innerhalb des gleichen Genres vorzieht, weil er selbst bei so genannten „freien“ (gebühren- oder werbefinanzierten) Angeboten letztlich mit seiner knappen Zeit bezahlen muss.

Allerdings kann weder zwischen Produktionsaufwand und Programmqualität noch zwischen Programmqualität und Rezipientennachfrage von einem linearen Zusammenhang ausgegangen werden. Zum einen gilt auch bei TV- und Radioangeboten das Gesetz abnehmender Grenzerträge: Die Qualität einer Live-Berichterstattung lässt sich beispielsweise ab einem gewissen Punkt durch zusätzliche Korrespondenten und Kameraperspektiven nicht mehr sichtbar steigern. Zum anderen gilt auch das Gesetz vom abnehmenden Grenznutzen bei den Rezipienten, d. h. eine weitere Erhöhung des Qualitätsstandards wird irgendwann einmal nicht mehr zu einer proportionalen Steigerung des Programmnutzens und damit des Marktanteils führen.

Im Zusammenhang mit der Analyse des Qualitätswettbewerbs bei Zeitungen wurde hierfür das Bild der „geknickten Nachfragekurve“ gewählt (Lacy/Simon, 1993: 31). Es gibt danach für Medienangebote einen Bereich, in dem die Kosten je Outputeinheit

Abbildung 1: Zusammenhang zwischen Qualität und Nachfrage nach Medieninhalten

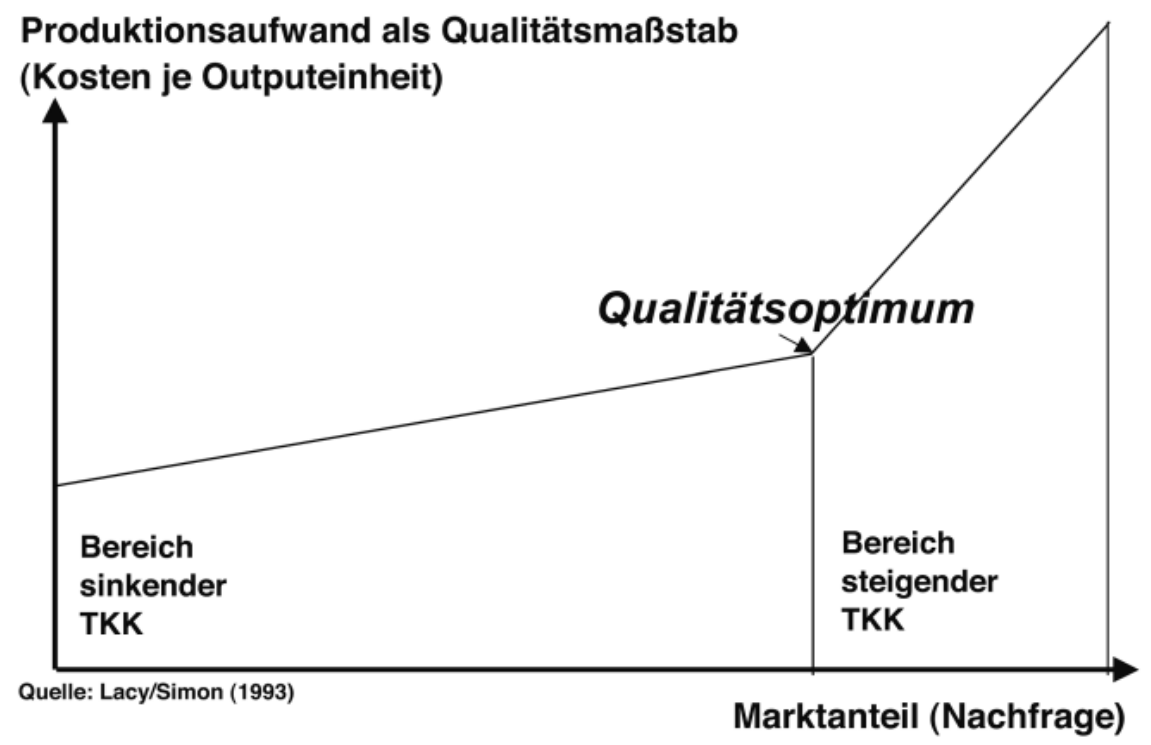


(Minutenkosten, Kosten je Seite etc.) durch „Qualitätsinvestitionen“ zwar steigen, die Tausenderkontaktkosten (TKK) aber fallen. Nach dem Überschreiten eines Qualitätsoptimums steigen dann bei Rundfunkanbietern mit den Minutenkosten auch wieder die TKK (vgl. Abb. 1).

\subsection{Genrespezifische Qualitäts-Nachfragefunktionen und TKK-Minimum}

Das über die Reaktion der Rezipientennachfrage auf Unterschiede im Produktionsaufwand definierte Qualitätsoptimum entspricht also dem Minimum einer U-förmigen Funktion, die die Höhe des TKK mit dem damit erzielbaren Marktanteil in Beziehung setzt. Diese soll im Folgenden als Qualitäts-Nachfragefunktion bezeichnet werden (Abb. 2).

Dabei ist davon auszugehen, dass das ökonomische Qualitätsoptimum bzw. das TKK-Minimum für verschiedene Programmgenres unterschiedlich hoch liegt und zudem mit unterschiedlichen Reichweiten bzw. Marktanteilen verbunden ist. Zum einen unterscheiden sich Genres aufgrund unterschiedlicher Produktionsprozesse generell in ihrem durchschnittlichen Produktionsaufwand. So sind im Fernsehen die Minutenkosten von Non-Fiktion-Programmen in der Regel niedriger als die von Fiktionprogrammen. Im Hörfunk sind sie für die Wortanteile in der Regel weit teurer als für die meist nicht selbst produzierten Musikanteile. Zum anderen gibt es für unterschiedliche Programmgenres unterschiedliche Reichweitenpotenziale. Gemessen an seinen Einschaltquoten ist beispielsweise das Marktsegment für Dokumentarfilme kleiner als das für Spielfilme. Das Reichweitenpotenzial für klassische Musik ist ebenfalls kleiner als das für Rock- und Pop-Musik. Deshalb ist auch anzunehmen, dass der Punkt, ab dem sich eine Qualitätssteigerung nicht mehr in einem überdurchschnittlichen Reichweitengewinn niederschlägt, für Programmgenres in kleineren Teilmärkten früher erreicht wird

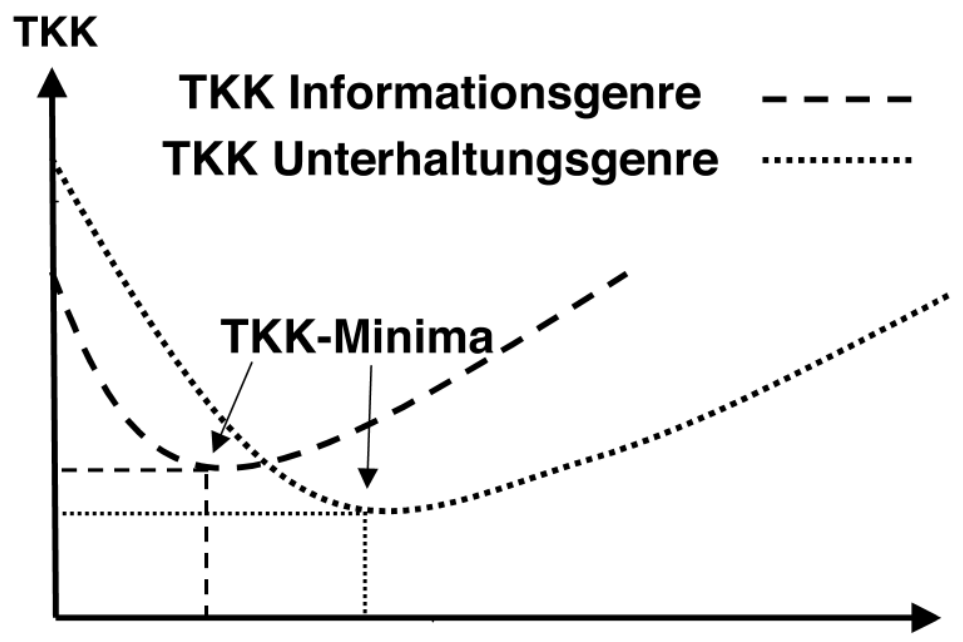


als in großen Teilmärkten. $\mathrm{Zu}$ vermuten sind also genrespezifische Qualitäts-Nachfragefunktionen mit unterschiedlichen TKK-Minima.

\subsection{TKK-Minimum und Wettbewerbsintensität}

Die Lage des TKK-Minimums, d. h. des Punktes auf der genrespezifischen QualitätsNachfragefunktion, der den in Bezug auf die Rezipientennachfrage jeweils kostenoptimalen Qualitätsstandard eines Genres definiert, wird außerdem von der Zahl der Wettbewerber bzw. der Intensität des Wettbewerbs zwischen diesen Anbietern beeinflusst. ${ }^{4}$ Je stärker der Qualitätswettbewerb, desto höher liegt die Kurve der Qualitäts-Nachfragefunktion und damit auch das TKK-Minimum.

Durch unterschiedliche Wettbewerbskonstellationen werden die Tausender-Kontakt-Kosten eines Genres zudem im Zeitablauf nicht konstant sein. Sie werden steigen, wenn sich durch Marktzutritte mehr Rundfunkveranstalter am Qualitätswettbewerb beteiligen. Bei sehr intensivem Qualitätswettbewerb bringt dann ein höherer Produktionsaufwand einem Anbieter im Vergleich zu den Vorjahren eventuell gar keinen Marktanteilsgewinn mehr, sondern er verhindert lediglich einen Marktanteilsverlust.

\section{Empirischer Zusammenhang zwischen Produktionsaufwand und Rezipientennachfrage im Jahr 2000}

\subsection{Empirische Grundlagen zur Bestimmung von Qualitäts-Nachfragefunktionen}

Als vorläufiges Fazit lässt sich festhalten, dass genrespezifische Tausenderkontaktkosten geeignete Kennziffern für Wirtschaftlichkeitsvergleiche zwischen Rundfunkanbietern sind, sofern zwei Bedingungen erfüllt sind: Zum einen muss bei Rundfunkangeboten - wie bei anderen Wirtschaftsgütern auch - ein unmittelbarer Zusammenhang zwischen dem Produktionsaufwand (gerechnet in Minutenkosten) und dem subjektiv wahrgenommenen Qualitätsstandard einzelner Sendungen existieren. Zum anderen muss ein höherer Qualitätsstandard auch zu positiven Nachfragereaktionen (höheren Reichweiten $^{5}$ ) führen. Dargestellt werden können solche empirischen Zusammenhänge zwischen Produktionsaufwand und Marktanteilen durch entsprechende Qualitäts-Nachfragefunktionen.

Die Datenlage erlaubt es nicht, diesen Zusammenhang für alle deutschen Programme und differenziert auf der Ebene von Programmgenres zu überprüfen. Für das Jahr 2000 lässt sich für TV-Vollprogramme und landesweite Hörfunkprogramme aber zumindest eine Analyse auf der Ebene der TKK für Gesamtprogramme durchführen.

Datengrundlage zum Kostenniveau und zum Anteil von Informations- bzw. Wortsendungen am jeweiligen Gesamtprogramm des Jahres 2000 sind für die öffentlichrechtlichen Anbieter die ARD- bzw. ZDF-Jahrbücher sowie für die privaten Anbieter die DLM-Erhebung zur wirtschaftlichen Lage des Rundfunks (DLM, 2002). Als Reichweitendaten werden die von der GfK für dieses Jahr gemessenen TV-Marktanteile bzw. die von der AG Media-Analyse ermittelten Hörerreichweiten der einzelnen Programme

4 Durch Absprachen bzw. abgestimmtes Verhalten in engen Oligopolen kann die Wettbewerbsintensität trotz hoher Anbieterzahl vergleichsweise gering sein.

5 Eine positive Nachfragereaktion kann sich bei gebührenfinanzierten (oder werbefinanzierten) Programmen nicht in einer höheren Zahlungsbereitschaft der Seher oder Hörer ausdrücken, sondern nur in einer erhöhten Nachfragemenge. 
zugrunde gelegt. Wegen der zugesagten Vertraulichkeit sind die Reichweitendaten der einzelnen privaten Anbieter als Indexwert aufbereitet, so dass keine Deanonymisierung möglich ist.

Die Rezipientennachfrage nach TV- und Radioprogrammen kann grundsätzlich in den unterschiedlichen Dimensionen Reichweite, Einschaltquote oder Marktanteil gemessen werden. Unter Reichweite wird im Folgenden die absolute Zahl von Zuschauern bzw. Hörern einer einzelnen Sendung verstanden. Unter Einschaltquote, die als Prozentwert dimensioniert ist, wird die Reichweite einer einzelnen Sendung in einem bestimmten Sendegebiet verstanden, die jeweils auf die Gesamtreichweite aller dort empfangbaren TV- oder Radioprogramme bezogen ist. Der Begriff Marktanteil (ebenfalls ein Prozentwert) wird häufig synonym zur Einschaltquote auch für Einzelsendungen verwendet. Im Folgenden wird er jedoch nur für das Gesamtprogramm eines bestimmten Anbieters genutzt.

\subsection{Bundesweite $T V$-Vollprogramme}

In die Untersuchung einbezogen wurden sechs private Vollprogramme (Kabel 1, Pro7, RTL, RTL 2, Sat.1 und VOX), die ARD- und ZDF-Hauptprogramme sowie - in einer Gesamtbetrachtung - alle Dritten Programme der ARD. Der durchschnittliche Produktionsaufwand reichte im Jahr 2000 von $320 €$ bis $3025 €$ je Sendeminute, die Sehdaueranteile lagen zwischen 2,8 \% und 19,3\%. Der Anteil der Informationssendungen am Gesamtprogramm (Selbstauskunft) betrug zwischen $12 \%$ und $46 \%$.

Eine lineare Regression, in der die Sehdaueranteile (Zuschauer ab 3 Jahre) als abhängige Variable und die Minutenkosten als unabhängige Variable definiert wurden, zeigt einen signifikanten und sehr engen Zusammenhang zwischen Produktionsaufwand und Marktanteil. Das korrigierte Bestimmtheitsmaß R²korr beträgt 0,926.

Nimmt man die jährlichen Produktionsaufwendungen je Marktanteilspunkt als Hilfsgröße für das jeweilige TKK-Niveau der neun TV-Vollprogramme, so reicht die Spanne von 30 Mio. $€$ bis 133 Mio. $€$ je Programm. Abbildung 3, in der die Sehdaueranteile in einen Indexwert (größter Marktanteil gleich 100) überführt wurden, zeigt zudem erwartungsgemäß, dass das TKK-Niveau mit dem Marktanteil steigt und dabei

\section{Abbildung 3: TKK-Nivean Fernsehen 2000 - bundesweite Vollprogramme Sebdaneranteil}

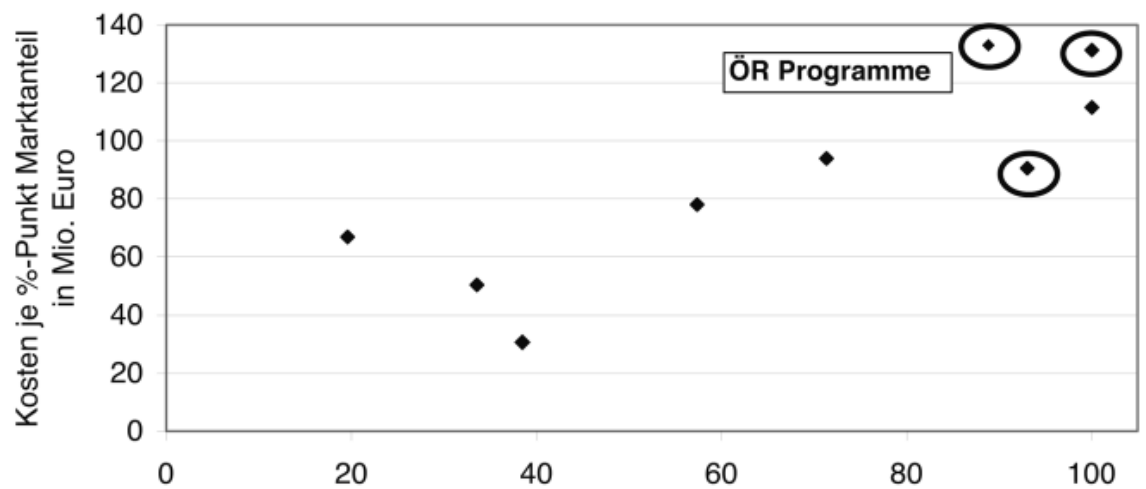

Sehdaueranteil (Index) 
Abbildung 4: TKK-Niveau Fernsehen 2000 - bundesweite Vollprogramme Informationsanteil

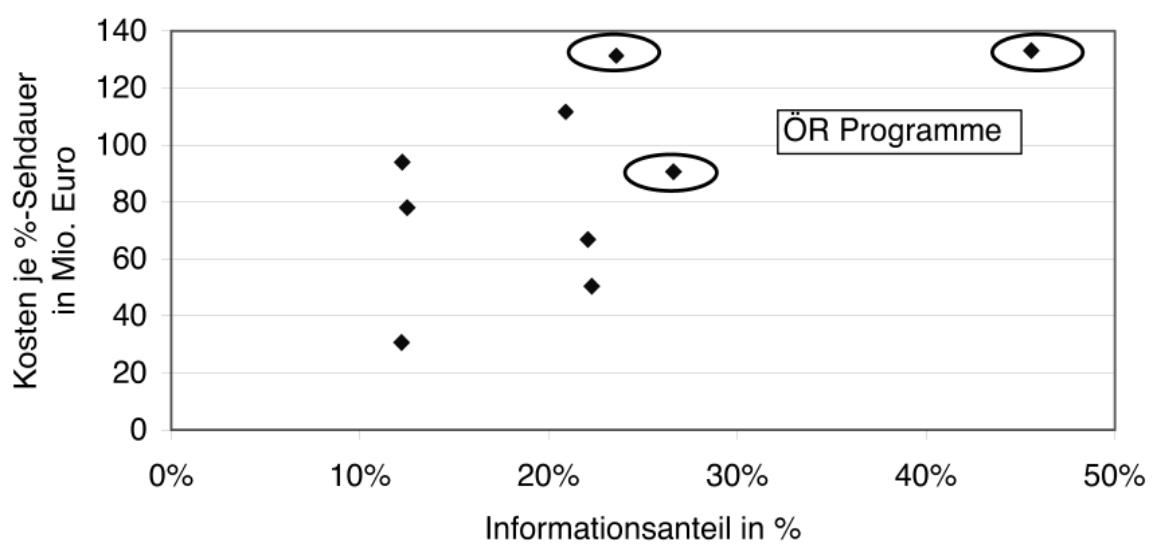

die öffentlich-rechtlichen Programme mit an der Spitze liegen. Aber auch bei privaten TV-Vollprogrammen haben die Anbieter mit den höheren Marktanteilen das höchste TKK-Niveau.

Gleichzeitig wird aus Abbildung 4 aber auch deutlich, dass dies gleichermaßen für den Informationsanteil am Gesamtprogramm gilt. Zu überprüfen wäre deshalb, in welchem Umfang dieses höhere TKK-Niveau der öffentlich-rechtlichen Programme auf ein geringeres Reichweitenpotenzial für Informations- als für Unterhaltungsprogramme zurückzuführen ist (vgl. unten Punkt 5).

\subsection{Landesweite Hörfunkprogramme}

In die Analyse einbezogen wurden die neun ARD-Anstalten, die im Jahr $2000 \mathrm{zu}-$ sammen 51 landesweit ausgestrahlte UKW-Hörfunkprogramme produziert haben, Deutschlandradio mit zwei bundesweit ausgestrahlten Programmen sowie 15 landesweite private Hörfunkprogramme, die in ihrem Sendegebiet unter den Privaten jeweils Marktführer waren. Die Minutenkosten lagen zwischen $7 €$ und $156 €$, die Reichweiten (Hörer gestern) zwischen 0,1 Mio. und 4,1 Mio. Hörern, wobei für die bis zu 7 Programme der einzelnen öffentlich-rechtlichen Anstalten jeweils die Gesamtreichweite in die Berechung einging. Der Wortanteil der Programme (Selbstauskunft) reichte von $10 \%$ bis $64 \%$.

Eine lineare Regression, in der die Hörerreichweiten als abhängige Variable und die Minutenkosten sowie der Wortanteil als unabhängige Variablen definiert wurden, zeigt keinen signifikanten Zusammenhang zwischen Produktionsaufwand und Marktanteil, aber einen engen Zusammenhang zwischen Produktionsaufwand und Wortanteil. Das korrigierte Bestimmtheitsmaß R²korr beträgt hier 0,673.

Berechnet man das Niveau der Tausenderkontaktkosten für die 25 Programme, reicht die Spanne von $11 €$ bis $923 €$ je Minute und 1000 Hörer. In Abbildung 5, in der die Reichweiten wiederum in einen Indexwert (größte Reichweite gleich 100) überführt wurden, wird sichtbar, dass dabei die öffentlich-rechtlichen Programme durchweg deutlich über dem TKK-Niveau der privaten Hörfunkprogramme liegen.

Abbildung 6 verdeutlicht jedoch, dass ihr Wortanteil ebenfalls über dem der meisten 
Abbildung 5: TKK-Niveau Hörfunk 2000 - ARD und private Erstanbieter - „Hörer gestern"

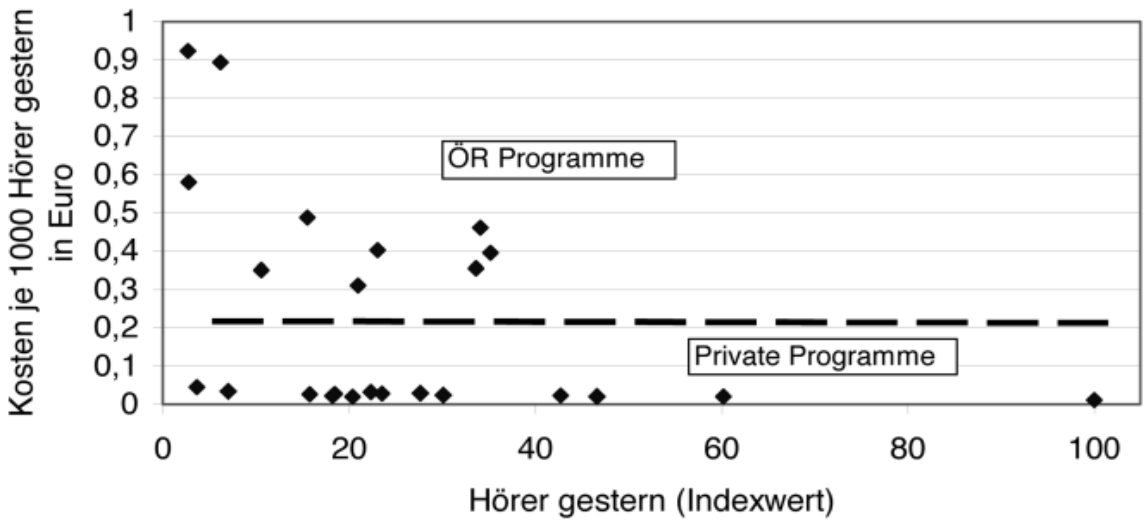

Privatangebote liegt. Auch hier wäre deshalb zu überprüfen, ob das höhere TKK-Niveau auf diese andere Programmstruktur der öffentlich-rechtlichen Angebote zurückzuführen ist - also entweder auf ein höheres Produktionskostenniveau für Wortprogramme oder auf ein geringeres Reichweitenpotenzial für „wortlastige“ Hörfunkprogramme.

Abbildung 6: TKK-Niveau Hörfunk 2000 - ARD und private Erstanbieter Wortanteil

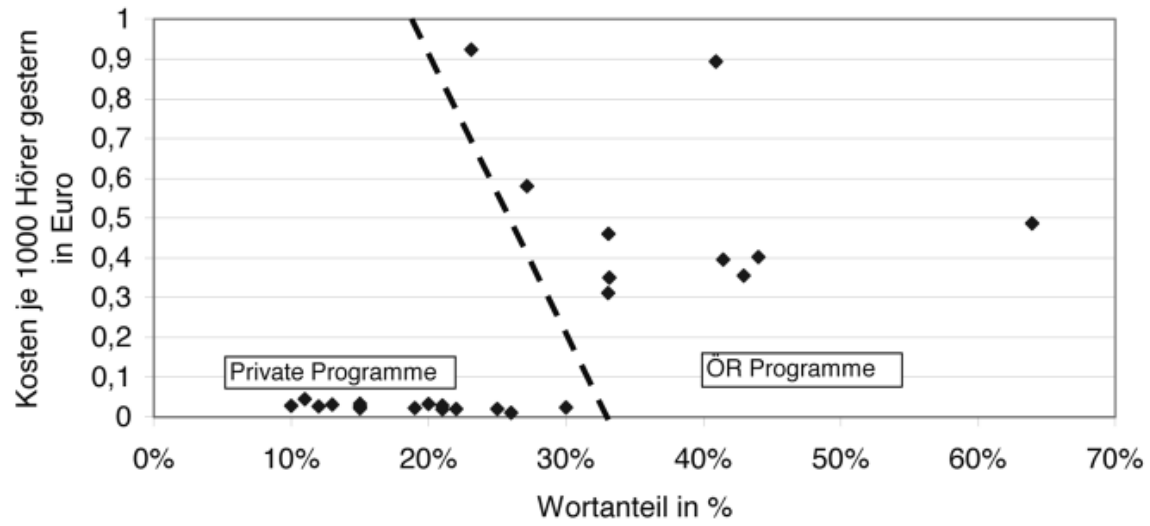

\section{Empirische Ergebnisse zum Einfluss der Programmstruktur auf das TKK- Niveau}

Welche möglichen Ursachen gibt es nun für die empirisch festgestellten Unterschiede im TKK-Niveau der einzelnen Programme? Die Kennziffer Tausenderkontaktkosten (TKK) ist der Quotient aus Minutenkosten (Dimension: € je Sendeminute) und Reichweite (Dimension: 1000 Zuschauer und Hörer) und kann für eine einzelne Sendung 
(Sendungsebene), als Durchschnitt für alle Sendungen eines bestimmten Programmgenres (Genreebene) oder als Durchschnitt für das Gesamtprogramm eines Anbieters (Programmebene) gebildet werden. Bei der Interpretation der Ergebnisse im Hinblick auf die Wirtschaftlichkeit von Rundfunkprogrammen sind also die Effekte durch potenzielle Einflussfaktoren auf der Ebene einzelner Pogrammgenres von denjenigen Effekten zu unterscheiden, die sich aus den jeweils unterschiedlichen Programmstrukturen der einzelnen TV- und Hörfunkprogramme ergeben.

\subsection{Einflussfaktoren auf das TKK-Niveau von Programmgenres}

Das TKK-Niveau eines Anbieters für ein Programmgenre wird von fünf wesentlichen Einflussfaktoren bestimmt (vgl. Abb. 7):

1. davon, ob die Produktionsprozesse optimal organisiert sind, einschließlich der Entscheidungen zwischen der Sendung von Eigen-, Auftrags- oder Fremdproduktionen (Kaufprogramme) (Faktor Produktionseffizienz).

2. davon, welches Qualitätsniveau das Unternehmen beim jeweiligen Genre anstrebt, einschließlich der Entscheidungen zwischen Erstsendungen und Wiederholungen (Faktor genrespezifischer Produktionsaufwand).

3. davon, welche Zahl an Wettbewerbern das eigene Marktanteilspotenzial beeinflusst; für einen Monopolisten liegt dieses Potenzial bei $100 \%$, bei 10 Anbietern beträgt es - sofern alle Wettbewerber qualitativ gleichwertige Programme senden - nur noch $10 \%$ (Faktor Anbieterzahl).

4. davon, welche Größe der jeweilige Genre-Teilmarkt hat (Faktor genrespezifisches Reichweitenpotenzial).

5. davon, wann das Genre überwiegend gesendet wird, da das Reichweitenpotenzial im Tagesverlauf unterschiedlich groß ist (Faktor Sendeplatz).

Der genrespezifische Produktionsaufwand hat also sowohl Einfluss auf die Minutenkosten (gemeinsam mit der Produktionseffizienz) als auch auf den Genre-Marktanteil des Anbieters (gemeinsam mit der Anbieterzabl). Mit welcher absoluten Zahl an Rezipienten (Reichweite) ein bestimmter Marktanteil verbunden ist, hängt außerdem zum einen vom genrespezifischen Reichweitenpotenzial und zum anderen von der Wahl des Sendeplatzes ab.

Abbildung 7: Einflussfaktoren auf das TKK-Niveau von Programmgenres

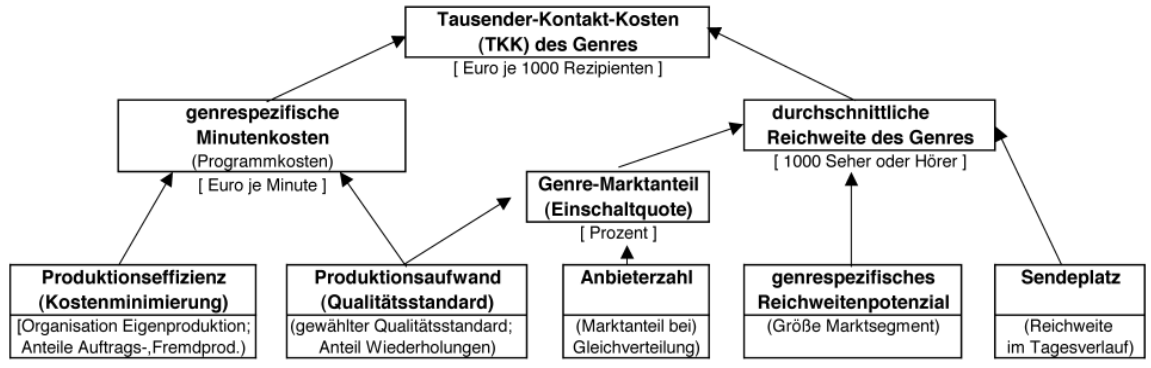




\subsection{Programmstruktureffekt und TKK-Niveau für Gesamtprogramme}

Auf der Ebene des Gesamtprogramms wird der durchschnittliche TKK zusätzlich durch einen Programmstruktureffekt beeinflusst, $d$. h. von den unterschiedlichen Anteilen einzelner Genres am Gesamtprogramm. Zwei Rechenbeispiele sollen diesen Effekt verdeutlichen:

\section{Beispielrechnung Programmstruktureffekt Fernsehen}

Unterstellt man, dass

- zwei Anbieter mit gleicher Produktionseffizienz und gleichem Produktionsaufwand, d. h. mit gleichen Minutenkosten arbeiten,

- diese Anbieter zwei Genres (Unterhaltung und Information) mit unterschiedlichen Programmanteilen (Anbieter A: $80 \mathrm{zu}$ 20, Anbieter B: $60 \mathrm{zu}$ 40) senden,

- die genrespezifischen Minutenkosten für Unterhaltungssendungen doppelt so hoch liegen wie für Informationssendungen und

- das genrespezifische Reichweitenpotenzial für Unterhaltungssendungen dreimal so hoch ist wie für Informationssendungen,

so ist im Ergebnis das TKK-Niveau für Informationssendungen rund 1,5-mal so hoch wie für Unterhaltungssendungen, und Anbieter B kommt auf ein fast $10 \%$ höheres TKK-Niveau als Anbieter A, wenn er den gleichen Marktanteil erreichen will (vgl. Tab. 1).

\section{Tabelle 1: Programmstruktureffekt Fernsehen}

\begin{tabular}{lccr}
\hline & Unterhaltung & Information & Gesamt \\
\hline (1) Index Minutenkosten & 100 & 50 & - \\
(2) Index Reichweitenpotenzial & 100 & 33,3 & - \\
(3) TKK-Niveau: $(1) /(2) * 100$ & 100 & 151,5 & - \\
(4) Programmstruktur Anbieter A & 0,8 & 0,2 & 1,0 \\
(5) TKK-Niveau Anbieter A: $(3) *(4)$ & 80 & $+30,3$ & $=110,3$ \\
(6) Programmstruktur Anbieter B & 0,6 & 0,4 & 1,0 \\
(7) TKK-Niveau Anbieter B: $(3) *(6)$ & 60 & $+60,6$ & $=120,6$ \\
$\quad$ Niveauunterschied B zu A: $(7) /(5)$ & & & 109,3 \\
\hline
\end{tabular}

\section{Programmstruktureffekt Hörfunk}

Unterstellt man, dass

- zwei Anbieter mit gleicher Produktionseffizienz und gleichem Produktionsaufwand, d. h. mit gleichen Minutenkosten arbeiten,

- diese Anbieter jeweils unterschiedliche Musik-Wort-Anteile senden (Anbieter C: 90 zu 10, Anbieter D: 70 zu 30),

- die Minutenkosten für Wortprogramme fünfmal so hoch sind wie für Musikprogramme und

- das Reichweitenpotenzial von Wortprogrammen bei der Hälfte der Musikprogramme liegt, 
so ist das TKK-Niveau für Wortprogrammanteile zehnmal so hoch wie das von Musikprogrammanteilen, und Anbieter D kommt auf ein um $95 \%$ höheres TKK-Niveau als Anbieter C, wenn er den gleichen Marktanteil erreichen will (vgl. Tab. 2).

Tabelle 2: Programmstruktureffekt Hörfunk

\begin{tabular}{lccc}
\hline & Unterhaltung & Information & Gesamt \\
\hline (1) Index Minutenkosten & 100 & 500 & - \\
(2) Index Reichweitenpotenzial & 100 & 50 & - \\
(3) TKK-Niveau: $(1) /(2) * 100$ & 100 & 1000 & - \\
(4) Programmstruktur Anbieter C & 0,9 & 0,1 & - \\
(5) TKK-Niveau Anbieter C: (3) $(4)$ & 90 & +100 & $=190$ \\
(6) Programmstruktur Anbieter D & 0,7 & 0,3 & - \\
(7) TKK-Niveau Anbieter D: $(3) *(6)$ & 70 & +300 & $=370$ \\
Niveauunterschied D zu C: $(7) /(5)$ & & & 194,5 \\
\hline
\end{tabular}

\subsection{Empirische Befunde zum genrespezifischen Produktionsaufwand und genrespezifischen Reichweitenpotenzial}

Es gibt wenig veröffentlichtes Datenmaterial zu den Minutenkosten und Reichweitenpotenzialen einzelner Programmgenres. Genrespezifische Minutenkosten werden, wie schon erwähnt, in den KEF-Berichten für alle öffentlich-rechtlichen Rundfunkanstalten veröffentlicht. In den Jahrbüchern der ARD werden auf Vollkostenbasis ebenfalls regelmäßig Durchschnittskosten für unterschiedliche TV- und Radioprogrammkategorien publiziert. Im Jahr 2000 (ARD-Jahrbuch, 2001, S. 369) lagen die Sendeminutenkosten für TV-Informationsprogramme im ARD-Hauptprogramm bei $1640 €$ (Kultur und Wissenschaft), $1820 €$ (Politik und Gesellschaft) bzw. $2730 €$ (Tagesschau/Tagesthemen). Die Spanne für Programme mit primär unterhaltendem Charakter war breiter, wobei das Niveau der Minutenkosten dabei durchweg über dem der Informationssendungen lag: $2920 €$ (Spielfilme), $4490 €$ (Non-fiktionale Unterhaltung), $4770 €$ (Musik), $8900 €$ (Sport), $12600 €$ (Fernsehspiel). Im Mittel hatten TV-Unterhaltungsangebote damit mehr als doppelt so hohe Minutenkosten wie Informations-, Bildungs- und Kulturprogramme.

Die ARD-Daten zeigen auch einen starken Unterschied im Produktionskostenniveau zwischen den Wort- und Musikanteilen der Radioprogramme. Die Minutenkosten für die drei Wortkategorien lagen im Jahr 2000 bei $50 €$ (Information und Service), $67 €$ (Unterhaltung) und $108 €$ (Kultur/Bildung). Dies war jeweils ein Vielfaches im Vergleich zu den Minutenkosten der Musikanteile, die bei $11 €$ (Rock/Pop) bzw. $23 €$ (Unterhaltungsmusik) lagen. Aus dem Rahmen fallen hier lediglich die $59 €$ für klassische Musik, die auf einen höheren Eigenproduktionsanteil für dieses Programmgenre (teilweise mit sendereigenen Orchestern) zurückzuführen sind.

Die in den obigen Beispielrechnungen zum Programmstruktureffekt getroffenen Annahmen zu den genrespezifischen Minutenkosten der unterschiedlichen TV-Programmanteile sind also der Größenordnung nach für die öffentlich-rechtlichen Anbieter nicht unrealistisch. Dies gilt auch für die unterstellten unterschiedlichen genrespezifischen Reichweitenpotenziale von TV-Informationssendungen und TV-Unterhaltungssendungen. Nach einer auf Basis der GfK-Programmkodierung - diese beruht auf Eigen- 
angaben der Veranstalter - vorgenommenen Auswertung war der Sehdaueranteil von Informationssendungen im Jahr 2004 nur etwa halb so hoch wie der gemeinsame Anteil von fiktionaler und non-fiktionaler Unterhaltung sowie von Sportprogrammen ( $\mathrm{Zu}$ bayr/Gerhard, 2005: 99).

Für Radioprogramme gibt es keine Nachfragedaten, bei denen nach Wort- und $\mathrm{Mu}$ sikanteilen getrennt wird. Dies mag unter anderem daran liegen, dass im Radio-Angebot der Typ der Magazinsendungen, in denen Musik- und Wortanteile gemischt werden, überwiegt. Radioprogramme unterscheiden sich deshalb eher über den Umfang ihres Wortanteils. Betrachtet man die Reichweiten der öffentlich-rechtlichen Informationsund Kulturprogramme mit hohem Wortanteil, so liegen diese weit unter denen der Magazin-Wellen. Die Annahmen der Beispielrechung, wonach für Radioprogramme mit einem hohen Wortanteil ein deutlich höheres TKK-Niveau nötig ist, um die gleichen Hörermarktanteile wie ein Radioprogramm mit überwiegendem Musikanteil zu erreichen, erscheinen deshalb ebenfalls nicht unrealistisch.

\section{Niveau der Tausenderkontaktkosten und öffentlich-rechtliche Qualitätsziele}

\subsection{Niedrigstes TKK-Nivean als Benchmark?}

Aufgrund des empirisch belegbaren positiven Zusammenhangs zwischen Programmaufwand und Rezipientennachfrage ergibt sich zunächst, dass Tausenderkontaktkosten ein geeigneter Indikator sind, um die Wirtschaftlichkeit sowohl zwischen privaten Anbietern von TV- und Radioprogrammen als auch zwischen öffentlich-rechtlichen Rundfunkanstalten zu vergleichen und dabei gleichzeitig die Unterschiede der von den Rezipienten gewünschten bzw. von den Aufsichtsorganen geforderten Programmqualität zu berücksichtigen. Das niedrigste TKK-Niveau eines Anbieters hätte dann jeweils zunächst nur eine Benchmark-Funktion (Schwertzel, 1997) für alle anderen Rundfunkanbieter des gleichen Typs im Hinblick auf die aktuell größtmögliche Produktionseffizienz.

Aufgrund der erläuterten Effekte unterschiedlicher Programmstrukturen auf das TKK-Niveau ist allerdings innerhalb beider Anbietertypen eine Orientierung an den Anbietern mit den jeweiligen niedrigsten genrespezifischen TKK-Niveaus sinnvoller als eine allgemeine Orientierung am Anbieter mit dem niedrigsten TKK-Niveau für das Gesamtprogramm.

Inwieweit kann nun aber ein niedriges genrespezifisches TKK-Niveau eines privaten Anbieters auch als Benchmark für öffentlich-rechtliche Rundfunkprogramme dienen? Die Antwort auf diese Frage hängt davon ab, ob man öffentlich-rechtliche Qualitätsstandards ausschließlich über Programmstrukturvorgaben operationalisiert, oder ob diese auch Marktanteilsziele als zusätzliche Dimension umfassen.

\subsection{Reichweite als Qualitätsdimension öffentlich-rechtlicher Programme}

Während man - sowohl was die objektive Messbarkeit der Qualität von Medieninhalten als auch was die Wahrnehmungsfähigkeit unterschiedlicher Qualitätsniveaus durch die Rezipienten angeht - in der medienökonomischen Literatur einer grundlegenden Skepsis begegnet (u. a. Heinrich, 1996; Gundlach, 1998; Lobigs 2004), gehört der Versuch, publizistische Qualität objektiv zu definieren, zu den zentralen Forschungsfeldern der Publizistik- und Kommunikationswissenschaft (u. a. Rager 1984; Schatz/Schulz, 1992; Ruß-Mohl, 1992; Wyss, 2002; Vowe/Wolling, 2004). Intern werden in den Rundfunkunternehmen für die Entwicklung von Qualitätskennziffern zudem Zuschauerbefragungen 
und organisatorische Maßnamen zur Sicherung eines angestrebten Qualitätsniveaus berücksichtigt (u. a. Buß/Gumbl, 2000; Zubayr/Darschin, 2003; Dintner/Brösel/Köcher, 2004; Anker, 2005; Blumers/Klingler, 2005; Tebert/Gierse, 2006).

Dabei besteht in der Kommunikationswissenschaft weitgehende Übereinstimmung, dass man die Qualität einzelner Medienangebote aus mindestens vier Perspektiven bewerten kann (Rosengren/Carlsson/Tagerud, 1991). Bei der Qualitätsdefinition kommen dann je nach Perspektive unterschiedliche Normensysteme zur Anwendung, die für verschiedene Bezugsebenen - Gesamtangebot aller Medien, Gesamtangebot eines Medienunternehmens oder Einzelbeitrag - operationalisiert werden (Wyss, 2002: 98) können. Diese vier Sichtweisen sind:

1. eine Reguliererperspektive, bei der die Erfüllung der gesellschaftlichen Informations- und Orientierungsfunktion im Vordergrund steht; Qualität bedeutet hier eine möglichst gute Abbildung der Realität in den Medieninhalten, die sich unter anderem in einem möglichst pluralistischen Angebot zeigt;

2. eine Kommunikatorperspektive, bei der die Erfüllung professioneller Standards im Vordergrund steht; die Qualität von Medieninhalten zeigt sich in diesem Fall unter anderem in einer möglichst großen Anerkennung durch Kollegen oder die Medienkritik;

3. eine Rezipientenperspektive, in der der subjektive Nutzen des einzelnen Mediennutzers im Vordergrund steht; die Qualität zeigt sich dann in der mehr oder weniger großen Akzeptanz der Medienangebote durch das Publikum. Diese Sichtweise entspricht also weitgehend der oben beschriebenen mikroökonomischen Interpretation von Qualität;

4. eine Wirkungsperspektive, bei der die vom Regulierer, Kommunikator oder Rezipienten intendierten kognitiven Kommunikationswirkungen im Vordergrund stehen; die Qualität von Medieninhalten zeigt sich danach in messbaren Veränderungen des Wissens, der Einstellungen oder des Verhaltens von Rezipienten.

Diese Systematisierung macht deutlich, dass die Qualität von Medien nicht über eine der vier Qualitätsdimensionen allein operationalisiert werden kann. Sinnvoll lassen sich Regulierer-, Kommunikator- oder Rezipientenperspektive jeweils nur zusammen mit einer zusätzlichen Wirkungsperspektive betrachten.

So sind beispielsweise Programmstrukturvorgaben, die Mindestanteile für Informations-, Bildungs- und Kulturinhalte im TV-Angebot oder für Wortelemente im Radio festlegen, als Operationalisierung von Qualität aus einer Reguliererperspektive plausibel. Dieses Ziel ist aber nicht hinreichend, wenn man die damit intendierten positiven Orientierungsleistungen für die Gesellschaft einbeziehen will. Sofern man einen gesellschaftlichen Informations- und Bildungsauftrag des öffentlich-rechtlichen Rundfunks einfordert, werden zwangsläufig auch Reichweitenziele als zusätzliche Qualitätsdimension relevant, da selbst das beste Angebot ohne Rezeption nicht die gewünschten Wirkungen erzielen kann.

\subsection{Reichweitenziele und TKK-Nivean}

Sofern aber das von öffentlich-rechtlichen Anbietern anzustrebende Qualitätsniveau sowohl über Programmstrukturziele (Anteil bestimmter Genres am Gesamtprogramm) als auch über Reichweitenziele (Marktanteilsvorgaben für die jeweiligen Genres) operationalisiert wird, hat dies Auswirkungen auf die mögliche Benchmark-Funktion privater Anbieter für öffentlich-rechtliche Angebote.

Höhere Tausenderkontaktkosten eines öffentlich-rechtlichen Anbieters im Vergleich 
zum niedrigsten TKK-Niveau können prinzipiell auf zwei Ursachen zurückzuführen sein:

- auf mangelnde Produktionseffizienz bzw. Wirtschaftlichkeit oder

- auf einen von diesem Anbieter gewählten höheren Qualitätsstandard des Outputs (und einen damit verbundenen höheren Produktionsaufwand).

Sofern es einen positiven Zusammenhang zwischen Qualität und Rezipientennachfrage gibt, muss sich dabei das höhere Qualitätsniveau in einem höheren Marktanteil des Anbieters niederschlagen. Sein Marktanteil wird also über dem rechnerischen Marktanteilspotenzial liegen, das bei einer rechnerischen Gleichverteilung der Marktanteile unter Berücksichtigung der Zahl aller Anbieter zu erwarten wäre. Umgekehrt gilt damit aber auch, dass Marktanteilsziele, die über dem Gleichverteilungsanteil liegen, zu einem höheren TKK-Niveau als dem genrespezifischen TKK-Minimum führen müssen (Abb. 8). Welches TKK-Niveau bei welchem Marktanteil angemessen ist, hängt vom Verlauf der Qualitäts-Nachfragefunktion für das entsprechende Programmgenre ab, die nur empirisch ermittelt werden kann.

Liegt das TKK-Niveau eines privaten Anbieters über dieser Funktionskurve, ließe sich entweder auf Produktionsineffizienz oder auf einen „zu hohen“ Qualitätsstandard schließen, der vom Rezipienten nicht mehr wahrgenommen bzw. nicht gewünscht wird.

Für einen öffentlich-rechtlichen Anbieter gelten jedoch in zweierlei Hinsicht andere Maßstäbe. Zum einen kann er als Non-Profit-Unternehmen durch den Gewinnverzicht - in der Größenordnung der branchentypischen Profitrate - generell alle Programmgenres zu einem höheren Qualitäts- und damit TKK-Niveau produzieren (Seufert, 2005: 372). Zum anderen muss er - unabhängig vom Programmstruktureffekt - immer dann

\section{Abbildung 8: Qualitäts-Nachfragefunktion und Produktionseffizienz bei Marktanteilsvorgaben}

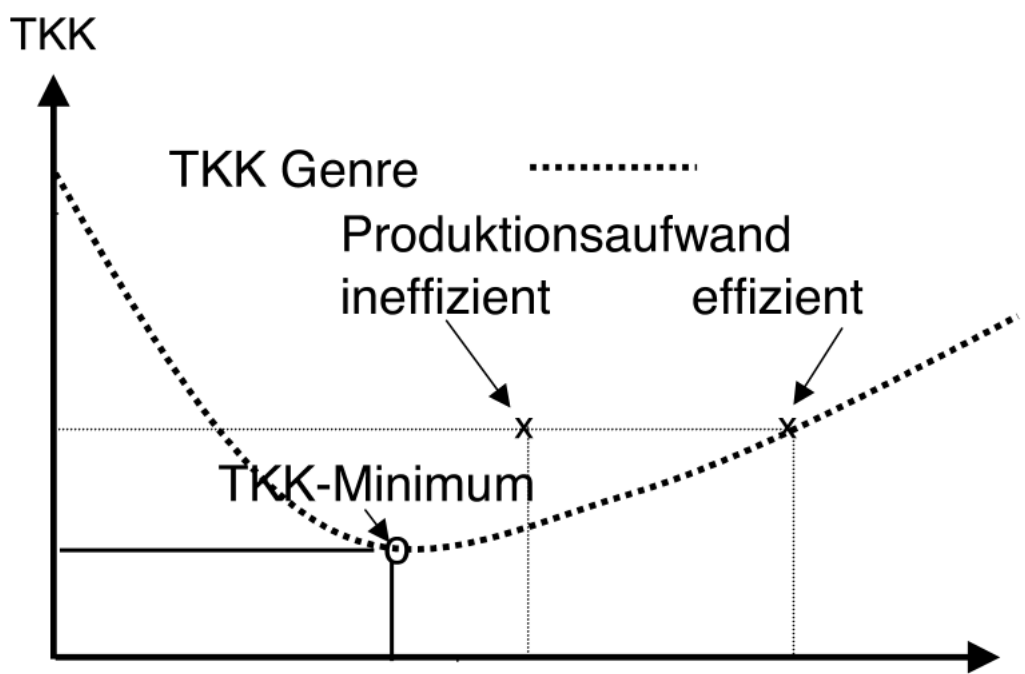

Marktanteile 
zu einem höheren genrespezifischen TKK-Niveau produzieren als der kostengünstigste private Anbieter, wenn für sein Programm Marktanteilsziele gelten, die über den bei gegebener Anbieterzahl zu erwartenden „Gleichgewichtsmarktanteilen“ liegen. Auch für öffentlich-rechtliche Anbieter gilt dabei, dass sich das für dieses Marktsanteilsziel angemessene TKK-Niveau aus den - empirisch zu schätzenden - Verläufen der Qualitäts-Nachfragefunktion für die einzelnen Programmgenres ergibt.

Im Hinblick auf die Eignung des kostengünstigsten TKK-Niveaus eines privaten Anbieters als Benchmark-Wert für öffentlich-rechtliche Angebote bedeutet dies also eine Einschränkung: Über diesem Niveau liegende genrespezifische TKK einer öffentlichrechtlichen Anstalt können erst dann als ein Indiz für Ineffizienz interpretiert werden, wenn sie bei Berücksichtigung der Marktanteilsziele dieser Anstalt nicht auf der Funktionskurve für das entsprechende Programmgenre liegen, sondern darüber.

\section{Resümee}

Der von der Kommission zur Ermittlung des Finanzbedarfs der Rundfunkanstalten (KEF) in ihren Wirtschaftlichkeitsvergleichen verwendete Indikator „durchschnittliche Minutenkosten“, der in der öffentlichen Debatte über die Effizienz der öffentlich-rechtlichen Anstalten zunehmend auch für Vergleiche mit privaten Anbietern herangezogen wird, führt zu Fehlinterpretationen. Implizit wird dabei von weitgehend standardisierbaren Produktionsprozessen ausgegangen, die die Heterogenität von TV- und Radioprogrammen (Unikatproduktion, Leistungsbündel aus unterschiedlichen Programmgenres) nicht beachten. Insbesondere wird damit aber auch der Zusammenhang zwischen Produktionsaufwand, Programmqualität und Rezipientennachfrage nicht beachtet. Tausenderkontaktkosten (TKK), die diesen Zusammenhang berücksichtigen, sind insofern auch für öffentlich-rechtliche Anbieter die geeigneteren Wirtschaftlichkeitsindikatoren.

Während allerdings private Anbieter ihre Programmstruktur so wählen können, dass sie ein möglichst geringes TKK-Niveau erreichen, unterliegen öffentlich-rechtliche Anstalten speziellen Qualitätsanforderungen, die sich auch in Programmstrukturvorgaben in Form von Mindestprogrammanteilen für Informations-, Bildungs- und Kultursendungen im TV-Programm oder Wortanteilen im Radioprogramm niederschlagen. Da diese Genres einen vergleichsweise hohen Produktionsaufwand (Radio-Wortprogramme) bzw. niedrigere Reichweitenpotenziale (TV-Informationsprogramme und RadioWortprogramme) haben, führt ein Programmstruktureffekt immer zu einem höheren TKK-Niveau für Gesamtprogramme, die einen hohen Anteil solcher Genres haben. Wirtschaftlichkeitsvergleiche zwischen verschiedenen Anbietern sollten deshalb nur auf Basis der TKK für Programmgenres durchgeführt werden.

Sofern man das TKK-Niveau privater Anbieter als Benchmark setzen will, müssen Reichweitenziele öffentlich-rechtlicher Anbieter für die einzelnen Programmgenres als zusätzliche Qualitätsanforderungen mit berücksichtigt werden. Je höher dieser angestrebte Marktanteil ist, desto stärker kann das genrespezifische TKK-Niveau der Rundfunkanstalt über dem des jeweils kostengünstigsten Anbieters liegen, ohne dass hieraus Unwirtschaftlichkeit abgeleitet werden kann.

Die Datenlage erlaubt es bislang nur, die Beziehungen zwischen dem Produktionsaufwand und Programmreichweiten auf der Ebene der Gesamtprogramme zu untersuchen und nicht für einzelne Programmgenres. Für Externe ist deshalb zurzeit auch keine angemessene Beurteilung der Wirtschaftlichkeit zwischen den öffentlich-rechtlichen Anstalten möglich. Voraussetzung wäre, dass die KEF zu den Minutenkosten der Genres - die noch stärker zu differenzieren wären - auch die damit korrespondieren- 
den Reichweitendaten erheben würde, so dass genrespezifische TKK berechnet werden können.

Eine solche Umstellung der KEF-Wirtschaftlichkeitsvergleiche auf den geeigneteren Indikator Tausenderkontaktkosten (TKK) hätte auch den Vorzug, dass in der öffentlichen Debatte über die Effizienz der öffentlich-rechtlichen Anbieter der Zusammenhang zwischen Kostenniveau und Qualität stärker beachtet würde als bisher, und dass dann wohl aus dem politischen Raum weniger untaugliche Sparvorschläge zu befürchten wären. Zudem verbesserten sich damit die externen Kontrollmöglichkeiten durch die Öffentlichkeit auch im Hinblick auf die Qualitätsziele der Rundfunkanstalten, da diese zu mehr Transparenz, insbesondere bei ihren Marktanteilszielen gezwungen würden.

\section{Literatur}

Anker, Heinrich (2005): Elemente einer integrierten Qualitätspolitik und Qualitätsstrategie öffentlich-rechtlicher Medien - Das Beispiel Schweizer Radio DRS. In: MedienWirtschaft Nr. $1 / 2005$, S. 25-39.

Blumers, Marianne/Klingler, Walter (2005): Fernsehprogramme und ihre Bewertung. Das ProgrammBewertungsVerfahren im SWR. In: Media Perspektiven 4/2005, S. 178-183.

Brenner, Christian (2002): Zur Gewährleistung des Funktionsauftrages durch den öffentlich-rechtlichen Rundfunk. Eine Konkretisierung der Aufgaben des öffentlich-rechtlichen Rundfunks im Fernseh-, Hörfunk- und Online-Bereich. Berlin: Tenea.

Buß, Michael/Gumbl, Harald (2000): Theoriegeleitete Evaluation im öffentlich-rechtlichen Rundfunk. Ein Konzept zur Qualitätsbewertung von Rundfunkangeboten. In: Media Perspektiven 5/2000, S. 194-200.

Czygan, Marco/Kallfaß, Herman H. (2003): Medien und Wettbewerbstheorie. In: Altmeppen, Klaus-Dieter/Karmasin, Matthias (Hrsg.): Medien und Ökonomie. Band 1/1. Wiesbaden: Westdeutscher, S. 283-304.

Dintner, Rolf/Brösel, Gerrit/Köcher, Anette (2004): Operative Controllingkennzahlen privater Fernsehsender. In: MedienWirtschaft Nr. 3/2004, S. 115-124.

DLM (Hrsg.) (2002): Beschäftigte und wirtschaftliche Lage des Rundfunks in Deutschland. 1999/2000. Berlin: Vistas. (Schriftenreihe der Landesmedienanstalten Band 24).

Eickhof, Norbert/Never, Henning (2000): Öffentlich-rechtlicher Rundfunk zwischen Anstaltsschutz und Wettbewerb. Universität Potsdam. Volkswirtschaftliche Diskussionsbeiträge, Nr. 35.

Eifert, Martin/Hoffmann-Riem, Wolfgang (1999): Die Entstehung und Ausgestaltung des dualen Rundfunksystems. In: Schwarzkopf, Dietrich (Hrsg.): Rundfunkpolitik in Deutschland. Wettbewerb und Öffentlichkeit. Band 1 und 2. München: dtv, S. 50-116.

Gemeinsame Initiative der Bundesländer Bayern, NRW und Sachsen zu einer Rundfunkstrukturreform (2003), dokumentiert unter: http://www.djv.de/downloads/initiative_bundeslaen der_rundfunkgebuehren.pdf; 27.7.2006.

Gundlach, Hardy (1998): Die öffentlich-rechtlichen Rundfunkanstalten zwischen öffentlichem Auftrag und marktwirtschaftlichen Wettbewerb. Berlin: S+W.

Hasebrink, Uwe (2005): Was hat die Quote im öffentlich-rechtlichen Rundfunk zu suchen? In: Ridder, Christa-Maria/Langenbucher, Wolfgang L./Saxer, Ulrich/Steininger, Christian (Hrsg.): Bausteine einer Theorie des öffentlich-rechtlichen Rundfunks. Festschrift für Marie-Luise Kiefer. Wiesbaden: VS, S. 450-454.

Heinrich, Jürgen (1996): Qualitätswettbewerb und/oder Kostenwettbewerb im Mediensektor? In: Rundfunk und Fernsehen 44, 2/1996, S. 165-184.

Heinrich, Jürgen (2005): Zur Funktionalität des Wettbewerbs im dualen System. In: Ridder, ChristaMaria/Langenbucher, Wolfgang L./Saxer, Ulrich/Steininger, Christian (Hrsg.): Bausteine einer Theorie des öffentlich-rechtlichen Rundfunks. Festschrift für Marie-Luise Kiefer. Wiesbaden: VS, S. 325-340. 
Heyen, Angelika (2001): Programmstrukturen und Informationsangebote im Radio. Ergebnisse und Erfahrungen aus sechs Jahren Programmforschung der TLM. In: Rössler, Patrick/Vowe, Gerhard/Henle, Victor (Hrsg.): Das Geräusch der Provinz - Radio in der Region. Festschrift 10 Jahre TLM. München: kopaed, S. 135-158.

Holznagel, Bernd/Vesting, Thomas (1999): Sparten- und Zielgruppenprogramme im öffentlichrechtlichen Rundfunk, insbesondere im Hörfunk. Baden-Baden: Nomos.

Kommission zur Ermittlung des Finanzbedarfs der Rundfunkanstalten (Hrsg.) (2003): 14. KEFBericht. Mainz: Selbstverlag.

Kommission zur Ermittlung des Finanzbedarfs der Rundfunkanstalten (Hrsg.) (2005): 15. KEFBericht. Mainz: Selbstverlag.

Kronberger Kreis (1989): Mehr Markt in Hörfunk und Fernsehen. Bad Homburg: Frankfurter Institut für Wirtschaftsordnung.

Krüger, Udo Michael (1991): Zur Konvergenz öffentlich-rechtlicher und privater Fernsehprogramme Entstehung und empirischer Gehalt einer Hypothese. In: Rundfunk und Fernsehen, 39. Jg., S. 83-96.

Kruse, Jörn (2004): Publizistische Vielfalt und Effizienz durch den Markt oder durch staatliche Regulierung. In: Friedrichsen, Mike/Seufert, Wolfgang (Hrsg.): Effiziente Medienregulierung. Marktdefizite oder Regulierungsdefizite? Baden-Baden: Nomos, S. 111-138.

Lacy, Stephen/Simon, Todd F. (1993): The Economics and Regulation of United States Newspapers. Norwood, NJ: Ablex.

Ladeur, Karl-Heinz (2000): Der „Funktionsauftrag“ des öffentlich-rechtlichen Rundfunks - auf „Integration“ festgelegt oder selbst definiert? In: Medien \& Kommunikationswissenschaft, 48. Jg., S. 93-106.

Lobigs, Frank (2004): Funktionsfähiger journalistischer Wettbewerb. Institutionsökonomische Herleitung einer fundamentalen publizistischen Institution. In: Siegert, Gabriele/Lobigs, Frank (Hrsg.): Zwischen Marktversagen und Medienvielfalt. Medienmärkte im Fokus neuer medienökonomischer Anwendungen. Baden-Baden: Nomos, S. 53-68.

Nafziger, Rolf/Schwertzel, Uwe (1996): Möglichkeiten und Grenzen von Kennziffern zur Beurteilung der Wirtschaftlichkeit öffentlich-rechtlicher Rundfunkanstalten. Köln: Institut für Rundfunkökonomie Uni Köln.

Picard, Robert G. (1989): Media Economics Concepts and Issues. Newbury Park [u.a.]: Sage.

Radke, Petra/Then Berg, Friedrich (2004): Neue Politische Ökonomie und Rundfunkregulierung - dargestellt am Beispiel öffentlich-rechtlicher Rundfunkanstalten. In: Friedrichsen, Mike/ Seufert, Wolfgang (Hrsg.): Effiziente Medienregulierung. Marktdefizite oder Regulierungsdefizite? Baden-Baden: Nomos, S. 139-156.

Rager, Günter (1984): Dimensionen der Qualität. Weg aus den allseitig offenen Richter-Skalen. In: Bentele, Günter/Hesse, Kurt R. (Hrsg.): Publizistik in der Gesellschaft. Festschrift für Manfred Rühl. Konstanz: Universitätsverlag, S. 189-210.

Rosengren, Karl E./Carlsson, Mats/Tagerud, Yael (1991): Quality in Programming. Views from the North. In: Studies of Broadcasting, Vol. 27, S. 21-80.

Ruß-Mohl, Stephan (1992): Qualitätssicherung im Journalismus. In: Publizistik, 37. Jg., S. 83-96.

Schatz, Heribert/Schulz, Winfried (1992): Qualität von Fernsehprogrammen. Kriterien und Methoden zur Beurteilung von Programmqualität im dualen Fernsehsystem. In: Media Perspektiven 11/1992, S. 690-712.

Schumann, Matthias/Hess, Thomas (2003): Grundfragen der Medienwirtschaft. Eine betriebswirtschaftliche Einführung. 2. Auflage, Berlin [u.a.]: Springer.

Schwertzel, Uwe (1997): Benchmarking für Rundfunkveranstalter: Konzeptionen für kennzahlengestützte Wirtschaftlichkeitsvergleiche von Anwendungsproblemen im Rundfunk. Berlin: Vistas.

Seufert, Wolfgang (1992): Die Entwicklung des Wettbewerbs auf den Hörfunk- und Fernsehmärkten in der Bundesrepublik Deutschland. Berlin: Duncker und Humblot.

Seufert, Wolfgang (2005): Öffentlich-rechtliche Rundfunkanstalten als Non-Profit-Unternehmen. In:Ridder, Christa-Maria/Langenbucher, Wolfgang L./Saxer, Ulrich/Steininger, Christian(Hrsg.): Bausteine einer Theorie des öffentlich-rechtlichen Rundfunks. Wiesbaden: VS, S. 367-379. 
Siegert, Gabriele/Brecheis, Dieter (2005): Werbung in der Medien- und Informationsgesellschaft. Eine kommunikationswissenschaftliche Einführung. Wiesbaden: VS Verlag.

Stock, Martin (2005): Duales System: funktionsgerecht ausgestaltet? In: Ridder, Christa-Maria/ Langenbucher, Wolfgang L./Saxer, Ulrich/Steininger, Christian (Hrsg.): Bausteine einer Theorie des öffentlich-rechtlichen Rundfunks. Wiesbaden: VS, S. 54-76.

Tebert, Miriam/Gierse, Christine (2006): Das Qualitäts-Controlling für das Erste. In: Media Perspektiven 1/2006, S. 23-31.

Vowe, Gerhard/Wolling, Jens (2004): Radioqualität - was die Hörer wollen und was die Sender bieten: vergleichende Untersuchung zu Qualitätsmerkmalen und Qualitätsbewertungen von Radioprogrammen in Thüringen, Sachsen-Anhalt und Hessen Magazin. München: Kopaed.

Wyss, Vinzenz (2002): Redaktionelles Qualitätsmanagement. Ziele, Normen, Ressourcen. Konstanz: UVK.

Zubayr, Camille/Darschin, Wolfgang (2003): Was leisten die Fernsehsender? Publikumsurteile über Fernsehprogramme in den Jahren 1993 bis 2002. In: Media Perspektiven 5/2003, S. 206-215.

Zubayr, Camille/Gerhard, Heinz (2005): Tendenzen im Zuschauerverhalten. Fernsehgewohnheiten und Fernsehreichweiten. In: Media Perspektiven 3/2005, S. 94-104. 